\title{
Analysis and Prediction of Student Evaluation Scores Based on Bias SVD
}

\author{
Rongrong Wang ${ }^{1}$, Yifan Zhu ${ }^{1}$, Sifan Zhang ${ }^{1}$, Qika Lin $^{2}$ and Zhendong Niu ${ }^{1+}$ \\ ${ }^{1}$ School of Computer Science and Technology, Beijing Institute of Technology, Beijing, China \\ ${ }^{2}$ School of Computer Science and Technology, Xi'an Jiaotong University, Xian, China
}

\begin{abstract}
Students' evaluation scores for teachers are significant indicators in the teaching evaluation process of a university or online course. There is a disadvantage in all existing evaluation methods, which regard students as the same and ignore the individual differences. To solve this problem, we propose a novel teaching evaluation method which is based on Bias SVD. Firstly, we convert the evaluation scores of teachers into a matrix. Then decompose this matrix by gradient descent and the biases of students in the evaluation process are iteratively obtained. By analyzing 63,193 evaluation records from 15 schools in Beijing Institute of Technology. We find that students who tend to give high scores have corresponding high offset values. We use a sentiment lexicon in the field of education to verify this method. By calculating emotion scores for teachers, we find that biases and scoring features are considerably correlative. Finally, we filtered the really too subjective scores through a certain threshold, and then used the XGBoost model to predict scores from the filtered data. It was shown that the combination method of Bias SVD and XGBoost can improve the accuracy of the prediction experimentally.
\end{abstract}

Keywords: evaluation analysis, individual differences, Bias SVD, gradient descent, score prediction.

\section{Introduction}

As a core component of education evaluation, student evaluation of teaching is crucial to teacher evaluation. A survey conducted by the German Presidents' Meeting [1] of German Universities [2] shows that 97\% of colleges and universities regularly conduct student evaluations.

\section{Related Work}

Although the role of student evaluation of teaching has been widely recognized, due to factors such as students' cognitive and biased behavior, the results of teaching evaluation are deviated from the actual situation. We propose using Bias SVD [3] when analyzing the impact of students' personal preferences. Bias SVD is commonly used in recommendation algorithms. Users have their peculiar preferences, for example, some users have a high score; and products have their own characteristics, such as the quality of the products. With this algorithm, user preferences and product characteristics can be taken into account. In this paper, a teaching evaluation analysis method based on deviation SVD is proposed. The algorithm can be used to analyze the characteristics of student scoring.To predict students' ratings of teachers, we can use machine learning methods. Xgboost [4]is a method based on GBDT [5].

\section{Methodology}

\subsection{BIAS SVD Gradient Descent Model}

In this paper, Bias SVD are applied to the decomposition of the teacher evaluation matrix. The input data of this paper's evaluation modeling is the processed student scoring matrix.

\footnotetext{
Corresponding author. Tel.: + (010-68915209);
}

E-mail address: zniu@bit.edu.cn. 
Modeling teacher's evaluation data through Bias SVD model:

$$
r_{u i}^{\wedge}=u+b_{u}+b_{i}+q_{i}^{T} P_{u}
$$

Where $r_{u i}^{\wedge}$ is the student's grade on the teacher's lecture, $u$ is the average of the scores, that is, the average score of the course score obtained by a teacher. $b_{u}$ reflects the student's offset, which is the personal trait that the student has when scoring. $b_{i}$ reflects the teacher's offset, which is the trait of the teacher's individual. $P_{u}$ is the student's feature matrix. $q_{i}$ is the teacher's feature matrix.

Then through iterative training, the values of these four parameters are converged with the loss getting smaller and smaller. Finally, we can get the student evaluation bias $\left(b_{u}\right)$ closest to the actual situation through training. We give the process of gradient descent, as shown below.

Algorithm: Bias SVD Iterative training algorithm

Input: Student's scoring matrix for teachers. This matrix is represented by mat

Output: The value of the four parameters during the gradient descent. Initialize four parameters $P_{u}, q_{i}$, $b_{u}, b_{i}$

for $\mathrm{i}=1 ; \mathrm{i} \leq$ mat. shape $[0] ; \mathrm{i}++$ do

$$
\begin{aligned}
e_{u i} & =r_{u i}-r_{u i} \\
\operatorname{SSE} & =\sum_{u j}\left(e_{u i}\right)^{2} \\
P_{u k} & =P_{u k}+\eta\left(e_{u i} q_{k i}-\lambda P_{u k}\right) \\
q_{k i} & =q_{k i}+\eta\left(e_{u i} p_{u k}-\lambda q_{k i}\right) \\
b_{i} & =b_{i}+\eta\left(e_{u i}-\lambda b_{i}\right) \\
b_{u} & =b_{u}+\eta\left(e_{u i}-\lambda b_{u}\right)
\end{aligned}
$$

end for

\subsection{Teaching Evaluation Prediction Model}

The traditional method of predicting scores is to directly bring each feature into XGBoost for modeling. But the existence of outliers in teaching evaluation usually seriously affects the quality of modeling and prediction, so it is necessary to eliminate the outliers in the data.

This paper proposes a method that uses Bias SVD to remove outliers. The explicit method is shown in the figure below.

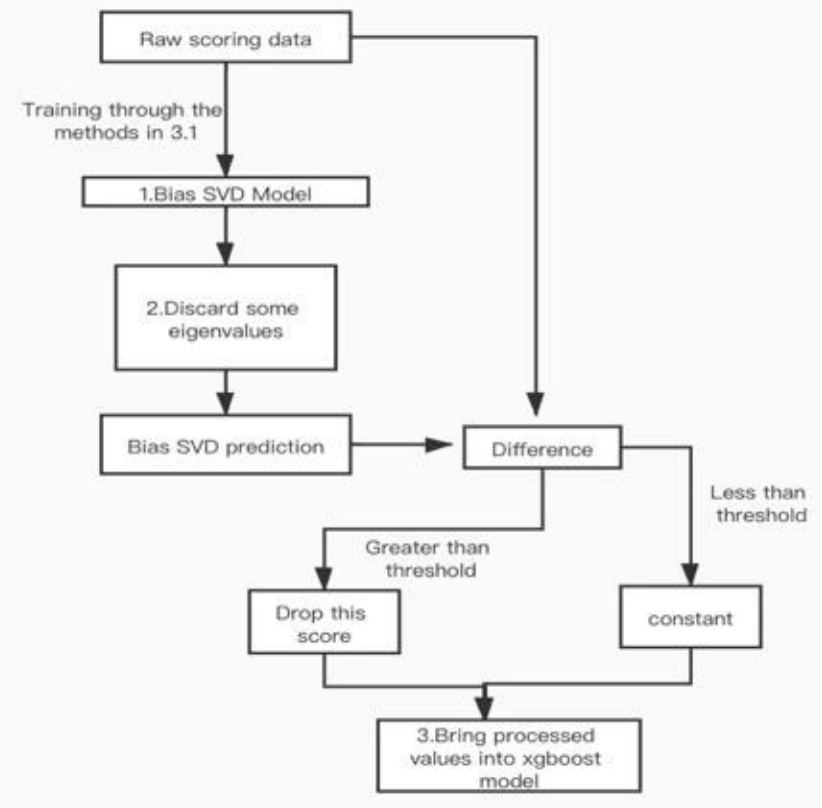

Fig. 1: Teaching evaluation prediction model 
The specific algorithm process is as follows:

1. Use the Bias SVD model to calculate the parameters.

The current input is $\left\{(s i, c i, s)_{1},(s i, c i, s)_{2}, \ldots,(s i, c i, s)_{n}\right\}$, the $s i$ is student id, the $c i$ is course id, the $s$ is score. The output is $b_{i}, b_{u}, u, q_{i}, p_{u}, r_{u i}^{\wedge}$.

2. Reconstruct the matrix and remove outliers.

a) Recalculate the score.

For the current input $(s i, c i, s)_{1}, p_{u}\left(\lambda_{0}, \lambda_{1}, \lambda_{2}, \ldots \lambda_{\min }, \ldots\right)$ find the smallest eigenvalue $\lambda_{\min }$ of the $\mathrm{p}_{\mathrm{u}}$ in the model and concatenate it to 0 to get a new matrix $p_{u}^{\prime}\left(\lambda_{0}, \lambda_{1}, \lambda_{2}, \ldots, 0, \ldots\right)$, use $r_{u i}^{\prime}=b_{i}+b_{u}+\mathrm{u}+q_{i}^{T} p_{u}^{\prime}$ recalculate the score.

b) Calculate the difference between the predicted and re-evaluated scores.

$\Delta=\left|\hat{\mathrm{r}}_{\mathrm{ui}}^{\prime}-\hat{\mathrm{r}}_{\mathrm{ui}}\right|$, set the threshold $\phi$, if $\Delta>\phi$, set the score at this place to 0 .

c) Iterate over all inputs, after executing a and $b$.

3. Re-extract eigenvalues based on the reconstructed matrix and put them in XGBoost.

\section{Experiment and Result}

The experiment is mainly divided into two processes: modeling by Bias SVD and score prediction. The data of this thesis come from the teacher evaluation system of Beijing Institute of Technology.

\subsection{Modeling by Bias SVD}

This paper conducts student evaluation analysis on the data of 15 schools such as the school of Materials, the school of Radio and TV, and the school of Computer. The following is a case study of the school of material, which explains the process of evaluation and modeling. It divides into four steps.

a) Data selection.

The material school's 1721 pieces of score and text comments are selected, including 423 students' evaluation of 139 teachers. In order to protect the privacy of the data, the teacher's name and the student's name are mapped to a number.

b) Convert rating data into a matrix.

Convert rating data into a matrix of $423 * 139$ dimensions. Matrix is represented by the list $[\mathrm{i}, \mathrm{j}]$. The horizontal axis represents the teacher. The vertical axis represents the student. For instance, list $(1,3,221)$ indicates that the first student scored 221 points for the third teacher, list $(1,2,0)$ indicates that the first student did not choose the second teacher's course or score the teacher.

c) Defining predicted values.

Use data triplet list $[i, j]$ to indicate the position of student and teacher in the scoring matrix. Use a list of values to represent the student's rating of the teacher. Modeling the evaluation matrix by the Bias SVD algorithm, calculate the predicted value.

d) Calculate the offset value.

The four parameters are trained by the gradient descent algorithm. Set the value of $\eta$ to be 0.005 and $\lambda$ to be 0.05 . $\mathrm{K}$ is the dimension of the feature matrix of $p_{u}$ and $q_{i}$, and Steps is the times of training. As the training round (steps) and the ratio of the training set to the test set (ratio) change, the root mean square error (RMSE) and mean square error (MSE) of the training set and test set are recorded respectively. We observed that when the $\mathrm{K}$ value is 7 , and the number of training rounds is 40 , the RMSE is the smallest. 
Table 1: Sample data during the gradient descent algorithm

\begin{tabular}{|c|c|c|c|c|c|c|}
\hline \multirow{2}{*}{$\boldsymbol{K}$} & \multirow{2}{*}{ Steps } & \multirow{2}{*}{ ratio } & \multicolumn{2}{|c|}{ RMSE } & \multicolumn{2}{|c|}{ MSE } \\
\cline { 4 - 7 } & & & Train Set & Train Set & Train Set & Test Set \\
\hline 10 & 50 & 0.9 & 1.0885 & 7.7161 & 0.4719 & 5.5401 \\
\hline 10 & 50 & 0.7 & 1.4004 & 7.7723 & 0.5558 & 5.3520 \\
\hline 9 & 50 & 0.7 & 1.4417 & 8.0617 & 0.6186 & 5.5024 \\
\hline 9 & 50 & 0.9 & 1.1428 & 7.7001 & 0.5144 & 5.7405 \\
\hline $\mathbf{7}$ & 40 & 0.9 & 2.6513 & 6.7016 & 1.6136 & 4.8368 \\
\hline
\end{tabular}

\subsection{Score Prediction}

As shown in the data preparation steps, the four tables are combined to obtain a master table. Based on the information in the master table, we can predict the student's rating of the teacher.

\subsubsection{Predictive Steps Using XGBoost Only}

It is mainly divided into three steps. Firstly, we augment the data based on the original data to obtain more features. we calculate the relevant information of each student, including the average score, maximum value, minimum value, course performance, and other related indicators of all courses.

\begin{tabular}{|l|r|r|r|r|r|r|r|}
\hline Stuld & grade & AllCount & CourselD & HeadCount & \multicolumn{2}{c|}{ Score } & \multicolumn{2}{c|}{ ClassCode Year } \\
\hline $\mathbf{2 1 2 0 1 6 1 1 3 3}$ & 78 & 210 & $402848815 \mathrm{ca} 1$ & 57 & 84.38 & 900012 & 2017 \\
\hline $\mathbf{2 1 2 0 1 6 1 1 3 6}$ & 79 & 162 & $402848815 \mathrm{ca} 1$ & 57 & 84.38 & 900012 & 2017 \\
\hline $\mathbf{2 1 2 0 1 6 1 1 3 6}$ & 87 & 206 & $402848815 \mathrm{ca} 1$ & 57 & 87.57 & 900017 & 2017 \\
\hline $\mathbf{2 1 2 0 1 6 1 1 3 6}$ & 86 & 206 & $402848815 \mathrm{ca} 1$ & 23 & 90.28 & 900010 & 2017 \\
\hline $\mathbf{2 1 2 0 1 6 1 1 3 6}$ & 93 & 214 & $402848815 \mathrm{ca} 1$ & 30 & 92.1 & 900030 & 2017 \\
\hline $\mathbf{2 1 2 0 1 6 1 1 3 6}$ & 81 & 218 & $402848815 \mathrm{ca} 1$ & 112 & 85.73 & 900001 & 2017 \\
\hline
\end{tabular}

\section{Data augmentation dimension}

\begin{tabular}{|c|c|c|c|c|c|c|c|c|c|c|c|}
\hline Stuld & MAX & MIN & AVG & grade & gradeProcess & AllCountAve & AllCount & HeadCount & Score & ClassCode & Year \\
\hline 2120161133 & 96 & 63 & 86.115 & 78 & 78 & 229 & 210 & 57 & 84.38 & 900012 & 2017 \\
\hline 2120161136 & 96 & 63 & $86.11 !$ & 79 & 79 & 221.2 & 162 & 57 & 84.38 & 900012 & 2017 \\
\hline 2120161136 & 95 & 79 & $86.93 t$ & 87 & 87 & 221.2 & 206 & 57 & 87.57 & 900017 & 2017 \\
\hline 2120161136 & 94 & 83 & 88.211 & 86 & 86 & 221.2 & 206 & 23 & 90.28 & 900010 & 2017 \\
\hline 2120161136 & 97 & 86 & 93.32 & 93 & 93 & 221.2 & 214 & 30 & 92.1 & 900030 & 2017 \\
\hline 2120161136 & 92 & 61 & $79.31 \%$ & 81 & 81 & 221.2 & 218 & 112 & 85.73 & 900001 & 2017 \\
\hline
\end{tabular}

Fig. 2: Examples of data augmentation

Then calculate the correlation coefficient between the features and the score. The features such as student number, course year, semester, course number, etc. have little correlation with the score. The average score, maximum value, and student achievement have great correlation with the score. Extract the first few columns with large correlation coefficients as the input features of the prediction model. Finally, the features are brought into the XGBoost model. It can be calculated that the mean absolute error is s 7.0893.

\subsubsection{Prediction Steps Using Bias SVD Model and XGBoost}

After calculating the parameters of Bias SVD through the algorithm of 3.2, take T $=40$ and $\phi=30$, then calculate the value of $\Delta$, see the table below for details. 
Table 2: Example of calculating the difference when taking 7 dimensions

\begin{tabular}{|c|c|c|c|c|c|c|c|c|c|c|}
\hline $\boldsymbol{b}_{\boldsymbol{u}}$ & $\boldsymbol{b}_{\boldsymbol{i}}$ & $\boldsymbol{q}_{\boldsymbol{i}}^{\boldsymbol{T}} \boldsymbol{p}_{\boldsymbol{u}}$ & $\boldsymbol{\Delta}$ & $\boldsymbol{p}_{\boldsymbol{u} 1}$ & $\boldsymbol{p}_{\boldsymbol{u} 2}$ & $\boldsymbol{p}_{\boldsymbol{u} 3}$ & $\boldsymbol{p}_{\boldsymbol{u} 4}$ & $\boldsymbol{p}_{\boldsymbol{u} 5}$ & $\boldsymbol{p}_{\boldsymbol{u} 6}$ & $\boldsymbol{p}_{\boldsymbol{u} 7}$ \\
\hline-1.21 & -1.73 & -87.07 & 40.37 & -4.53 & -2.67 & -0.7 & -0.69 & 0.85 & 2.38 & 3.12 \\
\hline-1.21 & -1.07 & -52.87 & 25.87 & -2.9 & -1.05 & -0.85 & -0.8 & -0.1 & 1.07 & 2.2 \\
\hline-1.21 & -1.19 & -60.72 & 27.13 & -3.05 & -1.15 & -0.46 & -0.24 & -0.05 & 1.86 & 2.84 \\
\hline
\end{tabular}

We analyzed the data which 50 points less than of the mean score in the original data. And we observed that the smaller eigenvalues of $\boldsymbol{p}_{\boldsymbol{u}}$ has a greater impact on the value of $q_{i}^{T} p_{u}$ (same to impact on the value of $\mathrm{r}_{\mathrm{ui}}$ ). The value of $\Delta$ can be understood as degree of positive evaluation and dissatisfaction with the teacher. For example, the $\boldsymbol{p}_{\boldsymbol{u}}$ of the selected data with indexes 17 and 22 is small, but the value of $\Delta$ at this position is big. The larger the value of $q_{i}^{T} p_{u}$, the more significant this effect. The value of $\Delta$ can be understood as degree of positive evaluation and dissatisfaction with the teacher. The value of $\Delta$ is larger, the degree of dissatisfaction is higher. If the score of the $\Delta$ is more than 30 points, the student's valuation is considered too arbitrary. After removing outliers, recalculate the relevant scoring information for each student and bring it into the XGBoost model. It can be calculated that the mean absolute error is 6.453 . We observed that after excluding outliers, the error of the score prediction is reduced.

\section{Conclusion}

This article mainly studies the differences between individual students in the process of teaching evaluation. We use the Bias SVD algorithm to quantify the differences between individual students. And by filtering outliers, the mean absolute error of the evaluation prediction was increased from 7.0893 to 6.453 .

The method in this article can quantify the deviation of students' evaluation of teaching. It has a beneficial effect on teaching evaluation. For example, the background of the teaching evaluation system can automatically analyze and record students with large deviations in teaching evaluation through the methods mentioned in the article, and can conduct a second survey on such students.Pupils who deliberately criticize teachers will be given certain punitive measures after verification by the school.

However, in the process of analyzing the deviations of the students 'teaching evaluation scores, we have not thoroughly studied the specific reasons behind the students' teaching evaluation deviations. We believe that in the later period, we should design a questionnaire for various factors that affect the deviation of students' evaluation, then investigate the root cause of the problem to properly guide students to evaluate teaching.

\section{Acknowledgment}

This work was supported by the National Key RI\&D Program of China (Grant number: 2019YFB1406302)

\section{References}

[1] Franke, Fabian, SühlStrohmenger, Wilfried. Teaching information literacy: the role of university libraries in Germany[J]. Journal of Information Literacy, 2015, 8(2):154-160.

[2] Marsh, H.W. and Roche, L.A. (1993), “The use of students' evaluations and an individually structured intervention to enhance university teaching effectiveness” , American Educational Research Journal, Vol. 30 No. 1, pp. 217-251.

[3] M. Aharon, M. Elad, A. Bruckstein. -SVD: An Algorithm for Designing Overcomplete Dictionaries for Sparse Representation[M]. IEEE Press, 2006.

[4] Tianqi Chen, Tong He, Michael Benesty. xgboost: Extreme Gradient Boosting[J]. 2016.

[5] Sakhnovich A. Nonisospectral integrable nonlinear equations with external potentials and their GBDT solutions[J]. Journal of Physics A Mathematical \& Theoretical, 2007, 41(15). 